\title{
The optical detection of retinal ganglion cell damage
}

\begin{abstract}
We provide an overview of developments in the use optical coherence tomography (OCT) imaging for the detection of retinal ganglion cell (RGC) damage in vivo that avoid use of any exogenous ligands to label cells. The method employs high-resolution OCT using broad spectral light sources to deliver axial resolution of under $5 \mu \mathrm{m}$. The resolution approximates that of cellular organelles, which undergo degenerative changes that progress to apoptosis as a result of axon damage. These degenerative changes are manifest as the loss of RGC dendrites and fragmentation of the subcellular network of organelles, in particular, the mitochondria that support dendritic structure. These changes can alter the light-scattering behavior of degenerating neurons. Using OCT imaging techniques to identify these signals in cultured neurons, we have demonstrated changes in cultured cells and in retinal explants. Pilot studies in human glaucoma suggest that similar changes are detectable in the clinical setting. Highresolution OCT can be used to detect optical scatter signals that derive from the RGC/inner plexiform layer and are associated with neuronal damage. These findings suggest that OCT instruments can be used to derive quantitative measurements of RGC damage. Critically, these signals can be detected at an early stage of RGC degeneration when cells could be protected or remodeled to support visual recovery.

Eye (2017) 31, 199-205; doi:10.1038/eye.2016.290; published online 6 January 2017
\end{abstract}

\section{Introduction}

Our understanding of retinal ganglion cell (RGC) death in diseases such as glaucoma and optic neuropathy has been transformed in recent years. ${ }^{1}$ For the most part, RGC death occurs by
JE Morgan, J Tribble, J Fergusson, N White and I Erchova

apoptosis, ${ }^{2}$ which is the culmination of a series of active cellular events involving organelle disruption. As with most chronic neuronal degenerative conditions these changes are preceded by a prolonged period of neuronal damage. In RGCs this is manifest as pruning of the processes (dendrites) that convey signals from outer retina to the RGC. ${ }^{3-6}$ Dendrites process and integrate signals from bipolar cells to provide the physiological basis for the receptive field of the RGC - the areas in the visual field, which when stimulated will activate a single RGC. The relationship between the integrity of the receptive field and the RGC dendritic tree has been explored in a number of studies. ${ }^{7}$

Animal models of glaucoma or optic neuropathy have shown a similar pattern of RGC degeneration in which peripheral dendrites are preferentially damaged. Dendrites that are closer to the cell body (primary and secondary dendrites) are preserved in late stages of disease and ${ }^{3,8}$ remain discernible in eyes with end stage RGC damage. ${ }^{9}$ While the majority of data showing these changes are based on rodent models of disease they are also seen in other glaucoma models such as cat ${ }^{10}$ and primate. ${ }^{3}$ Physiological studies in primates have shown that these early dendrite pruning are associated with reduced RGC sensitivity and contrast resolution. ${ }^{11}$

The presence of diseased but viable cells presents an attractive target for detecting early RGC damage. ${ }^{12}$ RGC dendrites provide a particularly sensitive readout of neuronal degeneration in cell and tissue based studies. Recent work in explant models of RGC degeneration has highlighted the possibility that these cells can be targeted to re-establish connections with the outer retina to provide a neural substrate for the restoration of vision in diseases such as glaucoma. ${ }^{12,13}$ The challenge is to determine whether these changes can be discerned using non-invasive techniques that are suitable for clinical application. This paper presents data from a number of studies based on
School of Optometry and Vision Sciences, Cardiff University, Maindy Road, Cardiff, UK

Correspondence:

JE Morgan, School of Optometry and Vision Sciences, Cardiff University, Maindy Road, Cardiff CF24 4HQ, UK Tel: +44 (0)29 20874374; Fax: +44 (0)2920874358. E-mail: morganje3@cardiff. ac.uk

Received: 24 October 2016 Accepted: 8 November 2016 Published online:

6 January 2017 
optical coherence tomography (OCT) imaging, which suggest that this many now be possible.

\section{Clinical imaging for the detection of retinal ganglion cell damage}

Imaging of the retinal and optic nerve has been transformed by the development of laser-based techniques that provide fast acquisition, high-resolution images of the eye. Two methods are of particular interest. Fluorescence imaging has shown particular promise for the in vivo detection of RGC degeneration ${ }^{14,15}$ but this remains restricted to the use of rodent models in which RGCs have been induced to fluoresce by genetic engineering. A more practical application of fluorescence imaging for the detection of RGC damage has used fluorescently tagged Annexin V antibody fragment to label cells that are in the early stages of the apoptotic pathway. ${ }^{16,17}$ The so-called DARC (detection of apoptosing retinal ganglion cells) technique has achieved notable success in animal models in which RGC apoptosis has been induced by axon crush by showing a clear increase in the numbers of tagged cells following axotomy. ${ }^{18}$ The technique is currently under evaluation to determine the rate of RGC apoptosis in clinical studies. The clinical utility of DARC will depend, to some extent, on precise quantification of the number of remaining RGCs. In experimental studies, the Annexin tag has been delivered by intravitreal injection: ${ }^{18}$ for clinical evaluation a topical delivery method would be ideal.

\section{Coherence based imaging}

OCT has become a staple clinical tool to image retinal layers in healthy and diseased tissue. The resolution of OCT instruments now falls within the scale of degenerating cellular organelles $(<5 \mathrm{um})^{19}$ and possibly neuronal activity. ${ }^{20}$ In the pre-apoptotic stages (Figure 1), mitochondrial networks fragment, preceding the nuclear disintegration which marks an irreversible commitment to cell death. ${ }^{21}$ Although nuclear fragmentation ${ }^{22}$ generates significant light scatter it could only be used as an index of cell death rather than disease. By contrast, mitochondrial fragmentation is a conveniently early feature of apoptosis which could be used to highlight regions where cells are diseased but not fully committed to cell death. Work in other areas- for example scatter dependent cell sorting has suggested that changes on this scale can generate detectable levels of optical scatter. ${ }^{23}$ Low coherence imaging methods have also been developed for the in vivo detection of dysplastic cellular changes most likely as a result of changes to the lightscattering properties of cell nuclei. ${ }^{24}$ We have previously reported mitochondrial changes in the dendritic trees of

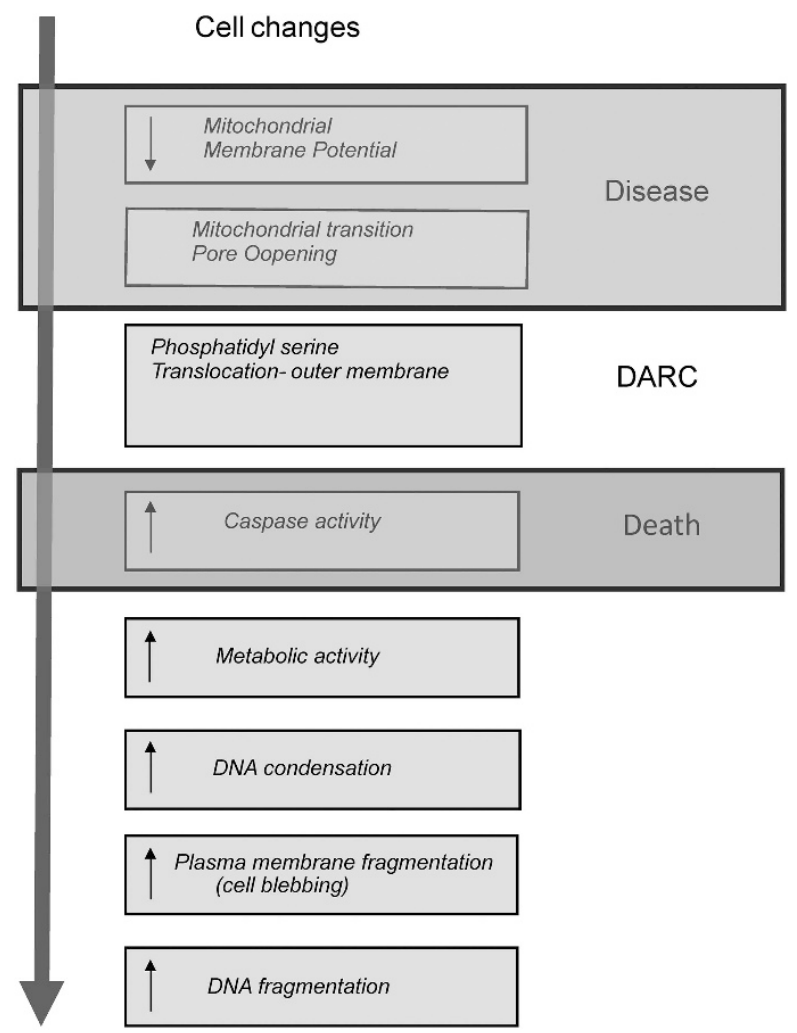

Figure 1 Overview of the sequence of cellular changes that precede apoptotic (programmed) cell death. 'Disease' covers changes that are reversible. 'Death' covers the point at which the cells commit to cell death. DARC, detection of apoptosing retinal ganglion cells.

mice with experimental (OPA1) related optic neuropathy. ${ }^{25}$ Early analysis of change in the mitochondrial distributions in the dendrites of midget cells in human glaucoma suggests that fragmentation and aggregation may offer feasible optical targets to generate changes in optical scatter that could be detected using OCT (Figure 2). However, the mitochondria within these dendrites will alter their distribution as a function of disease ${ }^{26}$ to generate changes in optical (light) scatter that should fall within the resolution limit of high-resolution OCTs. It could reasonably be argued that the availability of such high resolutions with next-generation OCT devices would allow the delineation (and counting) of individual RGCs, which would be of greater clinical value. Unfortunately, detecting the contrast change that demarcates cell boundaries would require an order of magnitude increase in sensitivity and clinical studies would be severely impacted by eye movement artifacts.

\section{Resolution limits and definitions}

For a proof of principle, the application of these techniques in the laboratory setting is simplified because 

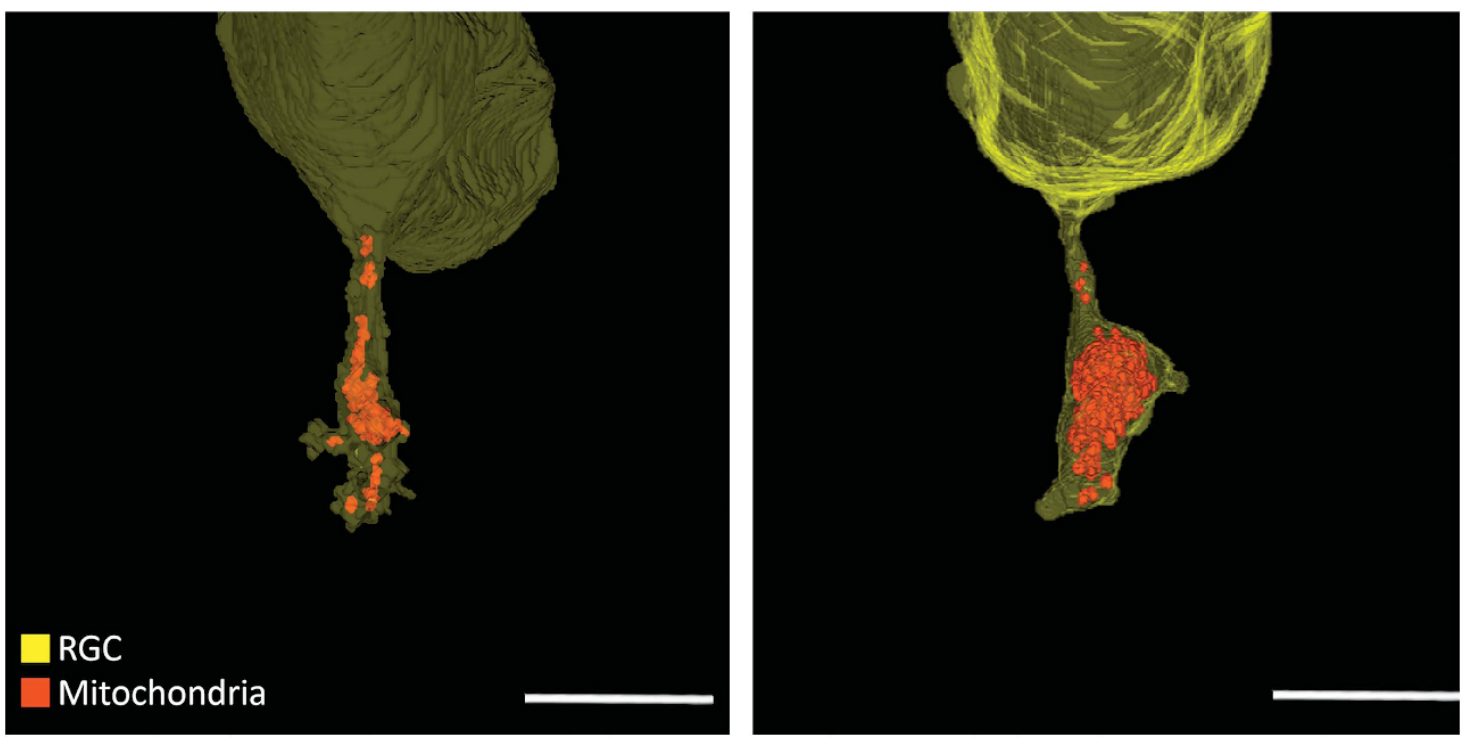

Figure 2 Serial block face scanning electron microscope reconstruction images of human midget RGC from a normal and glaucomatous eye. The processes in the glaucomatous eye are reduced and mitochondria show aggregation within the remaining dendrite structure. Each RGC serially sectioned at $100 \mathrm{~nm}$. Scale, $10 \mu \mathrm{m}$.

exposure times can be prolonged, sample movement minimized and the aperture and optics of the test system finely controlled. A key advantage of OCT imaging is that it decouples axial and transverse resolutions. ${ }^{27}$ In confocal imaging systems both are hostage to the aperture and aberrations of the eye. By contrast, with OCT, the axial resolution is limited by spectral spread of the light source. Although the terms 'laser' is often used to describe the illuminating beam in an OCT, the light sources are super luminescent diodes, which deliver a spread of wavelength, approximately centered on a midpoint (mean) wavelength. Clinically used OCTs typically use light with a mean wavelength in the range $800-1040 \mathrm{~nm}$ with a spectral spread of $50-80 \mathrm{~nm}$. The axial resolution is inversely related to the spectral spread with a theoretical axial resolution of 5-10 $\mu \mathrm{m}$ for these parameters.

$l_{\mathrm{C}}=\frac{2 \ln 2}{\pi} \frac{\bar{\lambda}^{2}}{\Delta \lambda}$

From Fercher et $\mathrm{l}^{27}$ ( $\lambda$, mean wavelength, $\Delta \lambda$ the spectral width).

It can be seen that an increase in the spectral spread $(\Delta \lambda)$, will reduce the minimum resolvable dimension $(l c)$, resulting in an increase in the resolution. For example, OCTs used for experimental work (eg, Phoenix Research Laboratories, Pleasanton, CA, USA) have a spectral spread of the order of $140 \mathrm{~nm}$, to provide a theoretical axial resolution of $\sim 3 \mu \mathrm{m}$. Until recently, cost has been a limiting factor for the use of wider spectral light sources (eg, Titanium Sapphire lasers). The wider availability of multiplexed diode light sources whose wavelength output can be approximated to a Gaussian distribution with a large spectral spread $(>100 \mathrm{~nm})$ will help in bringing higher resolution OCT into the clinical domain. When the resolution is matched in transverse and axial directions it is called isotropic-the ideal state for an imaging device. For most OCTs the axial resolution exceeds the transverse resolution since the latter is affected by aberrations of the human eye. These can be overcome using adaptive optics to yield high-resolution isotropic resolutions. ${ }^{28}$ These OCT systems are however, complex and with limited views of the retina and optic nerve which can limit clinical application.

\section{Light scatter during neuronal degeneration}

Since OCTs can potentially detect signals from objects approximating the dimension of subcellular organelles, we reasoned that they could be used to detect signals that correlate with the time course of neuronal degeneration. OCT images contain not only noise (speckle) arising from use of a laser as a light source but also from interference with multiple back scattering objects in a region of interest. The sources of speckle in OCT imaging has been covered in depth by Schmitt et a ${ }^{29}$ who usefully differentiated speckle into that containing information relevant to the imaged objects from speckle that degraded the image quality (Figure 3a). We therefore developed methods to extract useful measurements of optical texture from the speckle associated with OCT images. The signal to support this analysis combines optical scatter from changes in the tissue under examination, speckle due to 
a

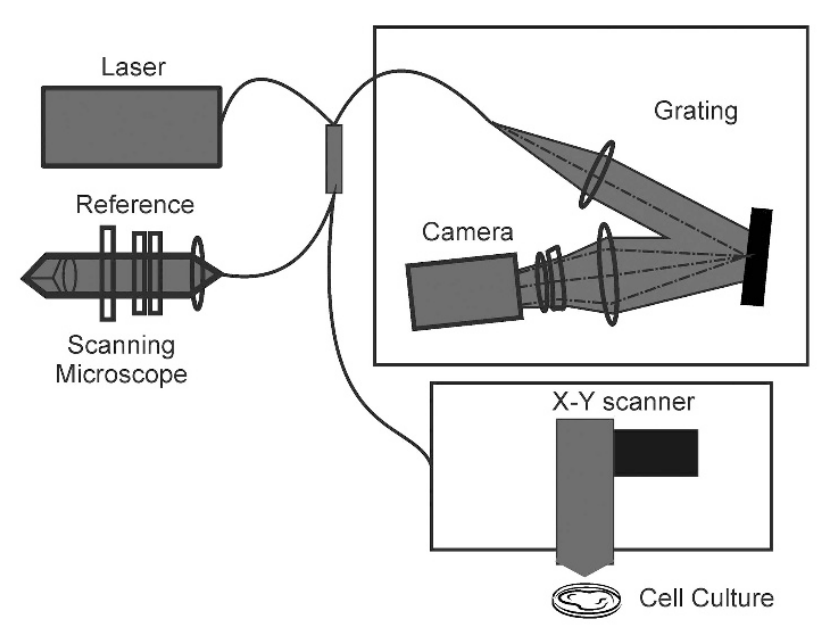

b

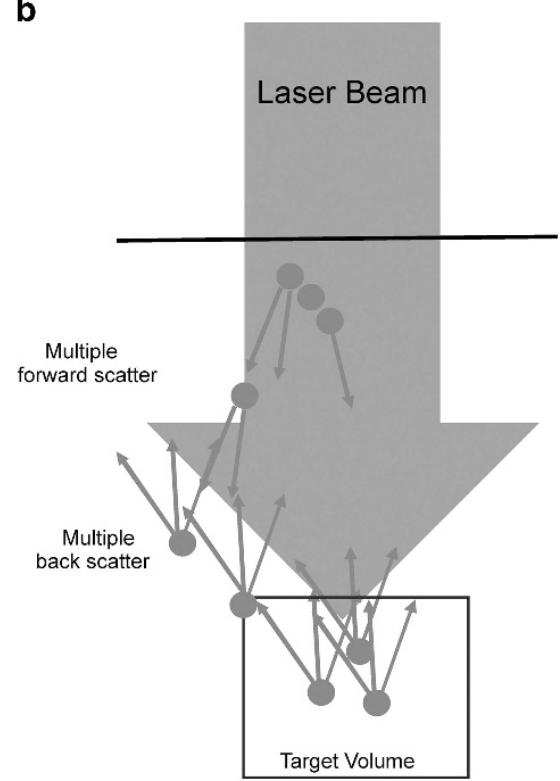

Figure 3 (a) Schematic representation of the OCT setup. Light source mean wavelength $800 \mathrm{~nm}$ (bandwidth $140 \mathrm{~nm}$ ). From Tudor et al. ${ }^{30}$. (b) Origin of scatter (speckle signal) shown the interaction of forward and back scattering of light to generate speckle. Adapted from Schmitt et al. ${ }^{29}$

forward light scatter and noise in the imaging system and detector. These signals are usually removed by image processing in commercial OCTs to facilitate segmentation of retinal boundaries.

We therefore constructed a custom OCT coupled to a standard compound microscope to examine changes in texture that occur in both cultured RGCs and retinal explants $^{30}$ (Figure 3b). The OCT used a Femtosecond Integral Ti:sapphire laser with a high bandwidth of $140 \mathrm{~nm}$ to provide a theoretical bandwidth of $3 \mu \mathrm{m}$. The examination of single cells over time allowed the detection of subcellular changes that occur once cell death has been initiated. We selected RGC5 cells which at the time of the study were thought to provide a useful mimic of RGCs. Subsequent analysis has shown that these are most likely a murine neuronal cell line, which can express some markers shared by RGCs when suitably induced. $^{31}$

\section{RGC5 neuronal degeneration over time}

Histological examination confirmed mitochondrial aggregation and caspase activation in RGC5 cell death following the initiation of apoptosis by the addition of staurosporine. ${ }^{30,32}$ The details of the mathematical modeling are described in details elsewhere. ${ }^{30}$ In brief, the image was parameterized using 65 features, which were then selected on the basis of the accuracy with which they correlated with culture stage. The selection of these dimensions was hypothesis free and based on the (unprocessed) spectral signal from the OCT. The aim of these pilot experiments was to determine if texture features could be detected that correlated with the stage of degeneration in the RGCs and allowed the effective discrimination of healthy from dying cells. Images were taken at regular intervals of the same region of interest, prior to RGC5 apoptosis (as demonstrated by Tunel staining).

We employed a Gaussian Mixture Model analysis to identify those variables related to image texture, which corresponded the degree of cellular degeneration. We aggregated these measures into a single dimensionless coefficient-the Texture Index (TI) to plot against time following the administration of staurosporine. These parameters were then fixed and used to analyze separate populations of RGC5 cells following the administration of staurosporine to induce apoptosis. We observed a progressive shift in the TI within $20 \mathrm{~min}$ of staurosporine, prior to the onset of significant apoptosis (Figure 4). These observations were consistent with a change in light scatter as a function of neuronal degeneration.

We next examined whether similar signals could be detected in in retinal explants. Explants provide an excellent model for the study of RGC degeneration since RGC degeneration is initiated by axotomy during specimen preparation. ${ }^{33}$ We have analyzed the changes in RGC dendritic structure using particle-mediated fluorescent labeling (Diolistics), showing that dendrite 


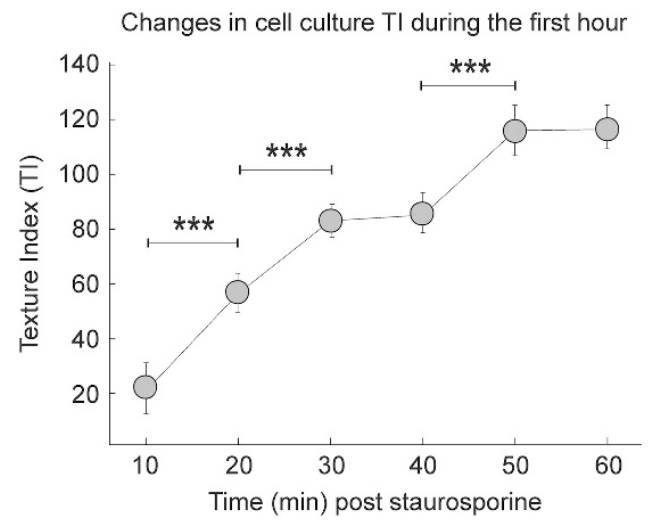

Figure 4 Gaussian Mixture Model analysis of early stages of apoptosis. Cell classification was based at each time point on the basis of texture analyses. A progressive shift in the Texture Index (TI) defined as before was observed within $20 \mathrm{~min}$ of the administration of staurosporine. Significance levels were calculated by $t$-test $\left({ }^{*} P, 0.05,{ }^{* *} P, 0.01,{ }^{* * *} P, 0.001\right)$. Adapted from Tudor et al. ${ }^{30}$

pruning occurs within $6 \mathrm{~h}$ of axotomy. These changes are shown in Figure 5 demonstrating that RGC dendrites are extensively pruned during the first 3 days of culture, ${ }^{13}$ supporting their use as a model of dendrite degeneration. For the purposes of this study, we randomly selected regions of interest from the inner plexiform layer (IPL) and subjected these to texture analysis using similar parameter settings as used for the RGC5 study. Retinal explants were scanned en face, RGC side up, and maintained their optical clarity throughout the experiment. The TI showed a monotonic increase as a function of time consistent with observations in single-cell data (Figure 6).

\section{Human studies}

Subsequent studies determined whether texture analysis could confer any benefit in the detection of glaucoma at an early stage in the disease. ${ }^{34}$ The experimental OCT device, which had a lower spectral spread and longer wavelength compared with the RGC5 study (NP photonics ASE light source, $1024 \mathrm{~nm}$, FWHM $80 \mathrm{~nm}$, theoretical axial resolution $7 \mu \mathrm{m}$ ), was connected to a clinical scanner and macular scans taken of patients with early/moderate glaucoma (14 normal, 10 glaucoma). As for the explant studies, texture signatures were extracted from the IPL but in addition these were passed to a Supported Vector Machine (SVM) for classification to determine if they could confer additional diagnostic precision in the discrimination of patients with early/ moderate glaucoma. Several image processing steps were required to eliminate artifacts arising from microsaccades. ${ }^{35} \mathrm{~A}$ small but potentially useful improvement in

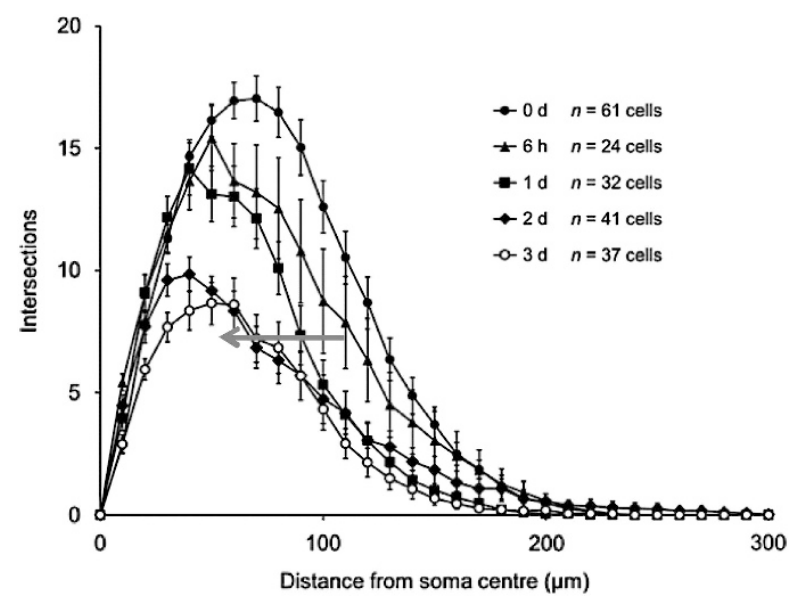

Figure 5 Sholl plots representing the complexity of RGC dendritic trees as a function of time in culture for murine retinal explants. The arrow indicates the leftward shift in the distributions as a function of time. Note that the first changes are seen within $6 \mathrm{~h}$ of culture. Adapted from Binley et al. ${ }^{13}$
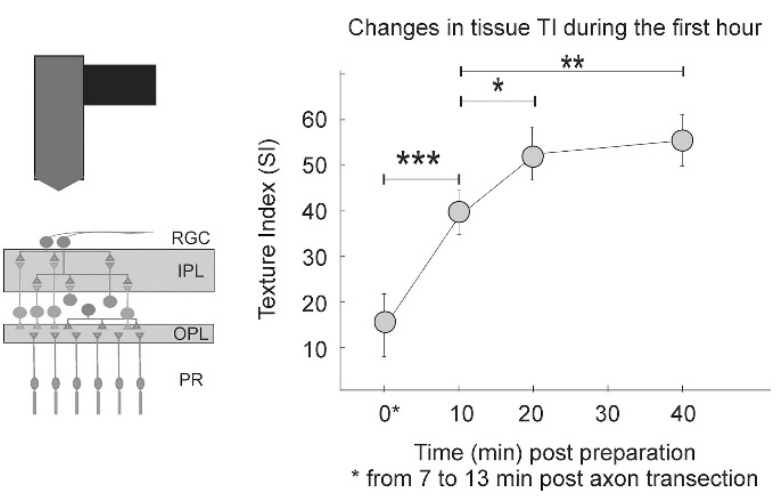

Figure 6 Changes in texture index as a function of time for murine retinas maintained in culture. OCT measurements were taken from the IPL within $1 \mathrm{~h}$ of explant preparation. The texture index shows a steady increase with time, changing as early as $10 \mathrm{~min}$ of culture time. The inset diagram shows the orientation of the OCT scanner and retinal explant. Adapted from Tudor et al. ${ }^{30}$

diagnostic accuracy (up to $4 \%$ ) could be seen when texture features were added to more conventional parameters used to discriminate glaucomatous eyes. ${ }^{34}$

\section{Texture analysis for the detection of retinal ganglion cell disease}

Our studies and the work of others support the use of texture analysis to quantify some dimensions of neuronal health taking RGCs as a specific example. The cell-based studies were performed with a highresolution OCT employing broad spectral light sources. Since the costs of these light sources have reduced 


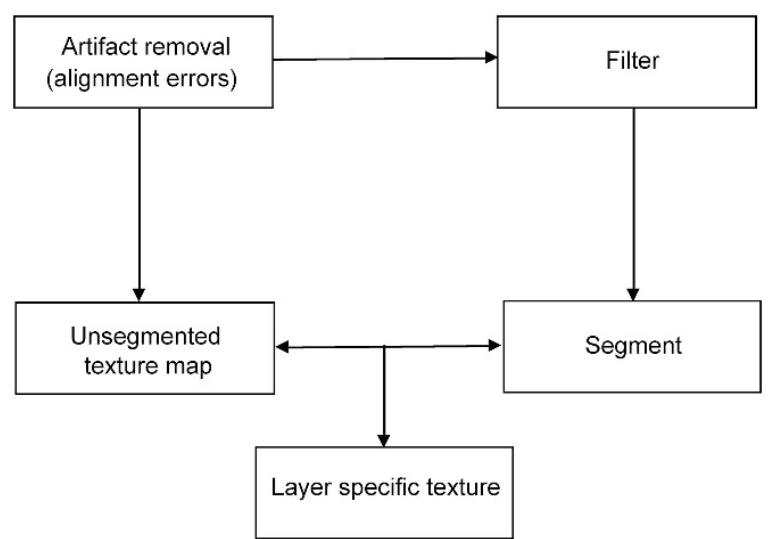

Figure 7 Scheme showing how OCT data can be separated into filtered data for the detection of retinal boundaries and unfiltered data for texture analysis. The two analyses can be merged to generate layer specific texture maps.

substantially, their use in the clinical setting is to be anticipated. All studies were conducted on custom OCTs in which all stages of image processing were known; this is not necessarily the case with commercially available devices, which could limit further clinical application of this approach.

Out data suggest that texture measurements and indices could be derived if OCT image filtering and segmentation were undertaken as suggested in Figure 7. In this model, unfiltered data are stored and processed to derive texture data in one analytic pathway and filtered data passed to another to segment retinal layers and define regions of interest. The texture scores can then be aligned to the segmented images to provide an index of neuronal health for selected retinal layers. In the context of studying RGC health, we suggest the IPL as an informative region of interest. It is clear that this technique has potential for the quantification of cellular health in other retinal layers, for example the photoreceptor layer for patient at risk of macular degeneration.

\section{Conflict of interest}

The authors declare no conflict of interest.

\section{Acknowledgements}

Funding was recieved from BBSRC UK, (BB/E017754/1), Fight for Sight (UK).

\section{References}

1 Almasieh M, Wilson AM, Morquette B, Cueva Vargas JL, Di Polo A. The molecular basis of RGC death in glaucoma. Prog Retin Eye Res 2012; 31(2): 152-181.
2 Kerrigan LA, Zack DJ, Quigley HA, Smith SD, Pease ME. TUNEL-positive ganglion cells in human primary openangle glaucoma. Arch Ophthalmol 1997; 115(8): 1031-1035.

3 Weber AJ, Kaufman PL, Hubbard WC. Morphology of single ganglion cells in the glaucomatous primate retina. Invest Ophthalmol Vis Sci 1998; 39(12): 2304-2320.

4 Morgan JE, Datta AV, Erichsen JT, Albon J, Boulton ME. Retinal ganglion cell remodelling in experimental glaucoma. Adv Exp Med Biol 2006; 572: 397-402.

5 Kalesnykas G, Oglesby EN, Zack DJ, Cone FE, Steinhart MR, Tian J et al. Retinal ganglion cell morphology after optic nerve crush and experimental glaucoma. Invest Ophthalmol Vis Sci 2012; 53(7): 3847-3857.

6 Williams PA, Howell GR, Barbay JM, Braine CE, Sousa GL, John SW et al. Retinal ganglion cell dendritic atrophy in DBA/2J glaucoma. PloS One 2013; 8(8): e72282.

7 Brown SP, He S, Masland RH. Receptive field microstructure and dendritic geometry of retinal ganglion cells. Neuron 2000; 27(2): 371-383.

8 Williams PA, Morgan JE, Votruba M. Opa1 deficiency in a mouse model of dominant optic atrophy leads to retinal ganglion cell dendropathy. Brain 2010; 133(10): 2942-2951.

9 Pavlidis M, Stupp T, Naskar R, Cengiz C, Thanos S. Retinal ganglion cells resistant to advanced glaucoma: A postmortem study of human retinas with the carbocyanine dye DiI. Invest Ophthalmol Vis Sci 2003; 44(12): 5196-5205.

10 Shou T, Liu J, Wang W, ZHOU Y. Differential dendritic shrinkage of $\alpha$ and $\beta$ retinal ganglion cells in cats with chronic glaucoma. Invest Ophthalmol Vis Sci 2003; 44(7): 3005-3010.

11 Weber AJ, Harman CD. Structure-function relations of parasol cells in the normal and glaucomatous primate retina. Invest Ophthalmol Vis Sci 2005; 46(9): 3197-3207.

12 Morgan JE. Retina ganglion cell degeneration in glaucoma: an opportunity missed? A review. Clin Exp Ophthalmol 2012; 40(4): 364-368.

13 Binley KE, Ng WS, Barde Y-A, Song B, Morgan JE. Brainderived neurotrophic factor prevents dendritic retraction of adult mouse retinal ganglion cells. Eur J Neurosci 2016; 44(3): 2028-2039.

14 Leung CK-S, Weinreb RN, Li ZW, Liu S, Lindsey JD, Choi N et al. Long-term in vivo imaging and measurement of dendritic shrinkage of retinal ganglion cells. Invest Ophthalmol Vis Sci 2011; 52(3): 1539-1547.

15 Leung CK-S, Lindsey JD, Crowston JG, Lijia C, Chiang S, Weinreb RN. Longitudinal profile of retinal ganglion cell damage after optic nerve crush with blue-light confocal scanning laser ophthalmoscopy. Invest Ophthalmol Vis Sci 2008; 49(11): 4898-4902.

16 Cordeiro MF, Guo L, Luong V, Harding G, Wang W, Jones HE et al. Real-time imaging of single nerve cell apoptosis in retinal neurodegeneration. Proc Natl Acad Sci USA 2004; 101(36): 13352-13356.

17 Cordeiro MF. DARC: a new method for detecting progressive neuronal death. Eye 2007, 21: S15-S17.

18 Guo L, Cordeiro MF. Assessment of neuroprotection in the retina with DARC. Prog Brain Res 2008; 173: 437-450.

19 Drexler W. State-of-the-art retinal optical coherence tomography. Prog Retin Eye Res 2008; 27(1): 45-88.

20 Bizheva K, Pflug R, Hermann B, Povazay B, Sattmann H, Qiu P et al. Optophysiology: depth-resolved probing of retinal physiology with functional ultrahigh-resolution 
optical coherence tomography. Proc Natl Acad Sci USA 2006; 103(13): 5066-5071.

21 Arnoult D. Mitochondrial fragmentation in apoptosis. Trends Cell Biol 2007; 17(1): 6-12.

22 Farhat G, Mariampillai A, Yang VXD, Czarnota GJ, Kolios MC. Optical coherence tomography speckle decorrelation for detecting cell death. SPIE 2011; 7907: 790710-790710-10.

23 Darzynkiewicz Z, Bruno S, Del Bino G, Gorczyca W, Hotz MA, Lassota P et al. Features of apoptotic cells measured by flow cytometry. Cytometry A 1992; 13(8): 795-808.

24 Wax A, Chalut KJ. Nuclear morphology measurements with angle-resolved low coherence interferometry for application to cell biology and early cancer detection. Anal Cell Pathol (Amst) 2011; 34(5): 207-222.

25 Williams PA, Piechota M, Ruhland von C, Taylor E, Morgan JE, Votruba M. Opa1 is essential for retinal ganglion cell synaptic architecture and connectivity. Brain 2012; 135(Pt 2): 493-505.

26 Mizielinska S, Frenguelli B, Harvey J. Mitochondrial dysfunction and dendritic beading during neuronal toxicity. J Biol Chem 2007; 282(36): 26235-26244.

27 Fercher AF, Drexler W, Hitzenberger CK, Lasser T. Optical coherence tomography - principles and applications. Rep Prog Phys 2003; 66(2): 239-303.

28 Torti C, Povazay B, Hofer B, Unterhuber A, Carroll J, Ahnelt PK et al. Adaptive optics optical coherence tomography at 120000 depth scans/s for non-invasive cellular phenotyping of the living human retina. Opt Express 2009; 17(22): 19382-19400.

29 Schmitt JM, Xiang SH, Yung KM. Speckle in Optical Coherence Tomography. J Biomed Opt 1999; 4(1): 95-105.

30 Tudor D, Kajić V, Rey S, Erchova I, Považay B, Hofer B et al. Non-invasive detection of early retinal neuronal degeneration by ultrahigh resolution optical coherence tomography. PLoS One 2014; 9(4): e93916.

31 Van Bergen NJ, Wood JPM, Chidlow G, Trounce IA, Casson RJ, Ju WK et al. Recharacterization of the RGC-5 retinal ganglion cell line. Invest Ophthalmol Vis Sci 2009; 50(9): 4267-4272.

32 Kim SC, Kang TJ. Texture classification and segmentation using wavelet packet frame and Gaussian mixture model. Pattern Recognit 2007; 40(4): 1207-1221.

33 Johnson TV, Martin KR. Development and characterization of an adult retinal explant organotypic tissue culture system as an in vitro intraocular stem cell transplantation model. Invest Ophthalmol Vis Sci 2008; 49(8): 3503-3512.

34 Anantrasirichai N, Achim A, Morgan JE, Erchova I, Nicholson L. SVM-based texture classification in Optical Coherence Tomography. IEEE 10th International Symposium on Biomedical Imaging (ISBI); 2013; 1332-1335.

35 Anantrasirichai N, Nicholson L, Morgan JE, Erchova I, Mortlock K, North RV et al. Adaptive-weighted bilateral filtering and other pre-processing techniques for optical coherence tomography. Comput Med Imaging Graph 2014; 38(6): 526-539. 